Review

\title{
The Varicella-Zoster virus IE4 protein: A conserved member of the herpesviral mRNA export factors family and a potential alternative target in antiherpetic therapies
}

\author{
Isabelle Ote, Jacques Piette, Catherine Sadzot-Delvaux* \\ Laboratory of Virology and Immunology, GIGA-Research, University of Liège (ULg), B-4000 Liège, Belgium
}

\section{A R T I C L E I N F O}

\section{Article history:}

Received 29 April 2010

Accepted 8 July 2010

\section{Keywords:}

VZV

IE4

Herpesviral homologues

mRNA export

Antiherpetic therapies

\begin{abstract}
A B S T R A C T
During a viral infection, in addition to cellular mRNAs, amounts of viral mRNAs have to be efficiently transported to the cytoplasm for translation. It is now established that herpesviruses encode a conserved gene family whose proteins act as viral mRNA export factors that mediate nucleocytoplasmic transport of viral transcripts and eventually modulate through this mechanism the antiviral response. This conserved family of proteins contains the IE4 protein of the Varicella-Zoster virus (VZV). Here, we compared the functional characteristics of IE4 with those of its herpesviral homologues and proposed a model by which IE4 would be able to recruit the essential TAP/NXF1 receptor to viral transcripts. Moreover, on the basis of their crucial roles in the infectious cycle, these conserved viral factors should be considered as alternative targets in therapeutic approaches. Here, we discussed the possibility of developing antiherpetic agents targeting IE4 or its herpesviral homologues.
\end{abstract}

(c) 2010 Elsevier Inc. All rights reserved.

\section{Contents}

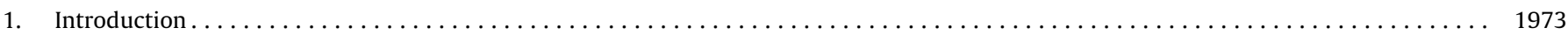

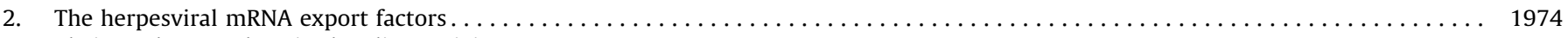

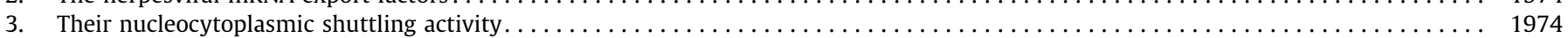

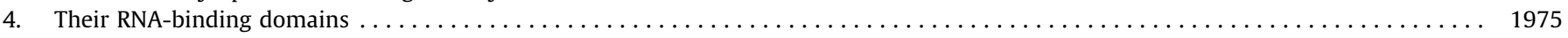

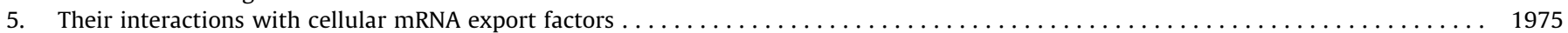

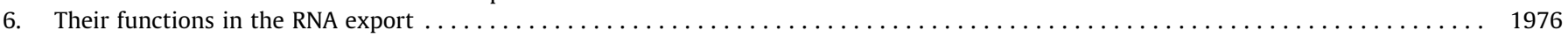

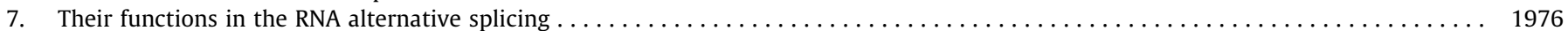

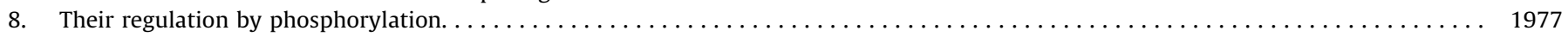

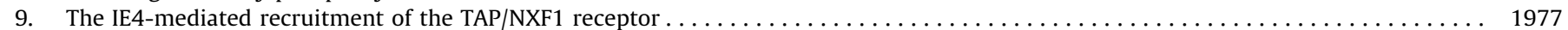

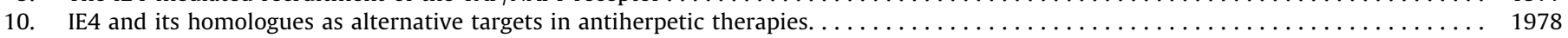

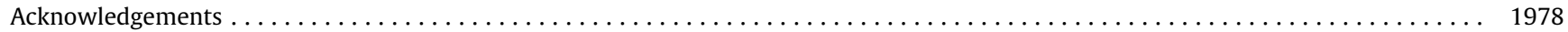

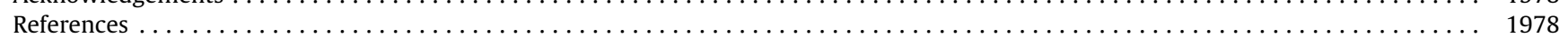

\section{Introduction}

Herpesviruses are DNA viruses whose genome is transcribed in three successive phases within the nucleus of the infected cells. Generally, in response to a viral infection, the host immune system reacts and sets up an inflammatory response aiming to eradicate the viral progression. Herpesviruses are known to induce such an innate immune response during acute infection. In parallel,

\footnotetext{
* Corresponding author. Tel.: +32 4366 2442; fax: +32 43662433.

E-mail addresses: csadzot@ulg.ac.be, jpiette@ulg.ac.be (C. Sadzot-Delvaux).
}

herpesviruses have also been described as being able to escape these antiviral responses. In this perspective, herpesviruses encode proteins that are known to interfere with the host immunity and whose expression was shown to be required to inhibit some antiviral responses. Among these proteins, a conserved family of herpesviral proteins has been described to interfere with the posttranscriptional gene regulation through the cellular machinery of mRNA splicing, stability, export and translation. This family contains the ICP27 protein of the herpes simplex virus type-1 (HSV-1), the UL69 protein of the human cytomegalovirus (HCMV), and the EB2 protein of the Epstein-Barr virus (EBV), respectively alpha-, beta- and gamma-herpesviruses. The IE4 protein, encoded 


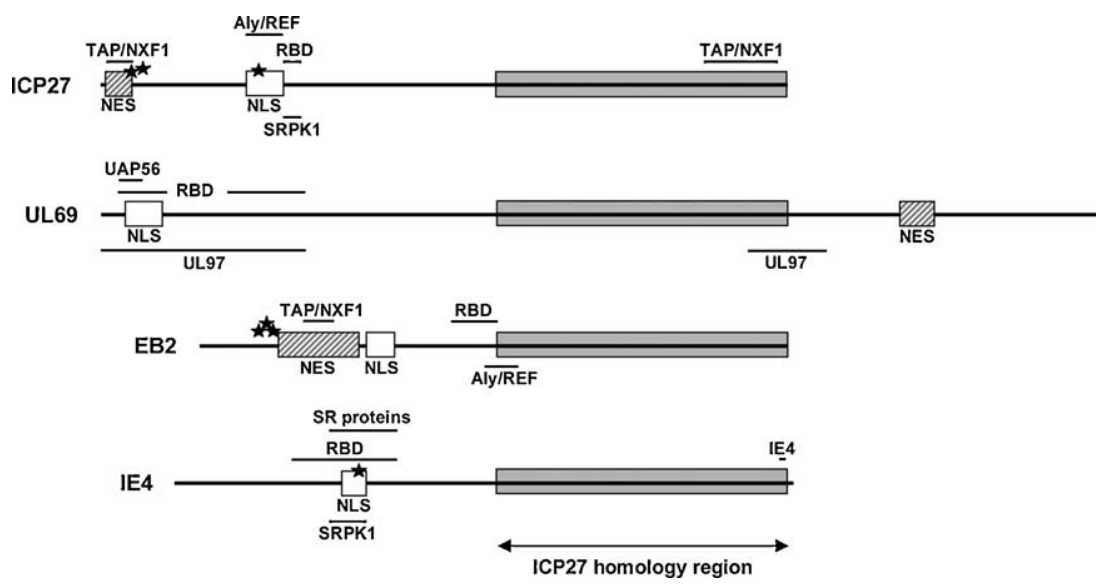

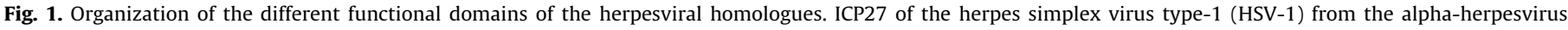

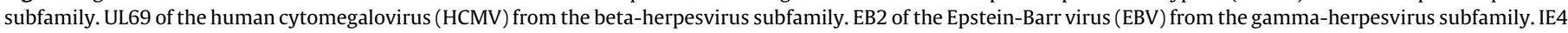

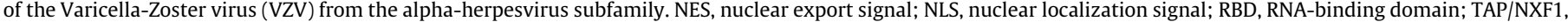

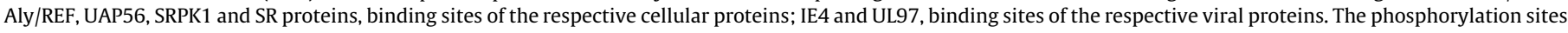
discussed in the text are shown by black stars.

by the Varicella-Zoster virus (VZV), another alpha-herpesvirus, also belongs to this conserved family and its characteristics will be compared in this review with those of its homologues.

\section{The herpesviral mRNA export factors}

Structurally, HSV-1 ICP27, HCMV UL69, EBV EB2 and VZV IE4 share an overall amino acid homology ranging between $20 \%$ and $30 \%$. Moreover, they all contain, within their C-terminal part, a homology region whose homology with ICP27 can be as high as 40-60\% (Fig. 1) [1,2]. Functionally, ICP27, UL69 and EB2 were all originally described as trans-regulatory proteins and it was initially assumed that their stimulatory functions on gene expression, from various viral as well as cellular promoters, occurred at the transcriptional level. Progressively, this transactivation appeared to occur at the RNA level, suggesting a posttranscriptional mechanism. Years later, it is now clearly demonstrated that these proteins share common characteristics, such as a nucleocytoplasmic shuttling activity, an RNA-binding domain (RBD) and the capacity to interact with cellular mRNA export factors, which identify them as viral mRNA export factors (Fig. 1). Available data suggested that IE4 also acts as an important regulator on VZV and cellular genes expression and could exert its functions at a post-transcriptional level. Although the molecular mechanisms are not yet fully characterized, we have recently shown that IE4 also shares the common characteristics of these herpesviral export factors [3].

\section{Their nucleocytoplasmic shuttling activity}

All members of this protein family have been reported to shuttle between the nucleus and the cytoplasm independently of virus-encoded cofactors indicating that it is an intrinsic characteristic [4-7]. Generally, nucleocytoplasmic protein trafficking occurs through direct interactions between the transport signals NLS and NES on the proteins and import or export receptors that mediate passage through the nuclear pore complex (NPC).

Nuclear localization signals (NLSs) have been described for the four homologues but they differ from each other (Fig. 2). First, ICP27 was shown to possess a strong NLS, mapping to residues 110-137, which bears similarity with the bipartite NLS found in Xenopus laevis nucleoplasmin. Moreover, the sequence mapping to residues $110-152$, including this strong NLS and the 15 contiguous residues which consist entirely of arginine and glycine residues, was demonstrated to function as nucleolar localization signal (NuLS) [8]. In contrast to ICP27, UL69 localizes within the nucleus but is excluded from the nucleolus [2]. It was also shown to contain an arginine-rich region at the N-terminus which resembles a bipartite NLS [6]. Similarly, EB2 was shown to contain two KH-rich motifs, mapping to residues $127-130$ and 143-145, which function as two independent NLS sequences [9]. A basic arginine-rich region, mapping to residues $130-143$, was also described in the $\mathrm{N}$ terminal part of IE4 and was proposed to be good candidate for NLS [7].

Even more different nuclear export signals (NESs) are present in the sequence of these viral proteins (Fig. 3). Most of the known shuttling proteins use a leucine-rich NES similar to that of HIV-1 REV, which directly interacts with CRM-1 nuclear export receptor

$\begin{array}{ll}\text { Nucleoplasmin } & \text { 154- KRPAATKKAGQAKKKK -171 } \\ \text { HSV-1 ICP27 } & \text { 109- ARRPSCSPERHGGKVARLQPPPTKAQPA -138 } \\ \text { HCMV UL69 } & \text { 18- ERERRARRARRFCLDYEPVPRKFRRERS }-47 \\ \text { EBV EB2 } & \text { 126- KRRRGEVHGCTDESYGKRR -146 } \\ \text { VZV IE4 } & \text { 129- RKHRDRSLSNRRRR -144 }\end{array}$

Fig. 2. Comparison of the different nuclear localization signals (NLSs) sequences The HSV-1 ICP27 NLS bears similarity with the bipartite Xenopus laevis nucleoplasmin NLS (underlined). The HCMV UL69 NLS is an arginine-rich (indicated in bold) region which resembles a bipartite NLS. The EBV EB2 NLS contains two independent KH-rich motifs (underlined). The VZV IE4 NLS was proposed to correspond to the basic arginine-rich (indicated in bold) region mapping to residues $130-143$. Numbers refer to the positions of the amino acid sequences within each protein.

$\begin{array}{lr}\text { HSV-1 ICP27 } & \text { 4- IDMLIDLGLDL -16 } \\ \text { HCMV UL69 } & \text { 596- APPAQPPSQPQQHYSEGELEEDEDSDDA -625 } \\ \text { EBV EB2 } & \text { 60- DEDPTPAHAIPARPSSVVITPTSASFVIPRKKW } \\ & \text { DLQDKTVTLHRSPLCRDEDEKEETGNSSYTR } \\ & \text { GHKRRRGEVHGCTDES -141 } \\ \text { VZV IE4 } & \text { 320- LLENLKLKLG -331 }\end{array}$

Fig. 3. Comparison of the different nuclear export signals (NESs) sequences. The HSV-1 ICP27 NES is a leucine-rich NES similar to the HIV-1 REV NES. The conserved amino acids are shown in bold. The HCMV UL69 NES is a 28-amino acid domain whose essential residues are indicated is bold. The EBV EB2 NES is a leucine-rich region mapping to residues 61-140. A leucine-rich motif found into the central part of VZV IE4 could correspond to an NES. Numbers refer to the positions of the amino acid sequences within each protein. 
to be transported into the cytoplasm. This CRM-1-dependent nuclear export can be blocked by the antibiotic leptomycin B (LMB), which prevents the association of NES-containing proteins with CRM-1 [10]. Whereas ICP27 and EB2 possess a leucine-rich NES located in their N-terminal part, the requirement of CRM-1 for their shuttling has been much discussed. Since initial studies suggested that a LMB-sensitive leucine-rich region could be involved in the nucleocytoplasmic shuttling of EB2 [5,11], later studies identified the unique sequence responsible for the EB2 shuttling that was shown to be LMB-insensitive [9,12]. Similarly, although the export of ICP27 was first reported to be sensitive to LMB [13,14], the leucinerich N-terminal sequence that is required for an efficient ICP27 export was further shown to function as a CRM-1-independent NES $[15,16]$. Singularly, a novel type of LMB-insensitive export signal was identified within the UL69 unique C-terminal region [6]. This 28amino acid domain is not a leucine-rich one and the export pathway used by this unique NES has not yet been defined. Whereas VZV IE4 exhibits a mainly cytoplasmic localization and should bear a nuclear export signal, no classical NES sequence can be found within its $\mathrm{N}-$ terminal region. However, a putative sequence rich in leucine residues can be seen into the central part of the protein and could correspond to an NES, but the functionality of this putative signal has never been demonstrated [7].

\section{Their RNA-binding domains}

Another characteristic of all known viral mRNA export factors is the presence of an RNA-binding domain (Fig. 4). ICP27 was shown to require an arginine-glycine-rich region, mapping to residues 138-152, that constitutes an RGG box RNA-binding domain [17]. Recently, three arginine residues within this RGG box were shown to be methylated in vivo but this methylation appears to regulate protein-protein interactions of ICP27 in the cytoplasm rather than RNA binding [18]. In contrast, EB2 was demonstrated to harbour an RNA-binding motif in a 33-amino acid segment that has the structural features of arginine-rich RNA-binding motifs (ARMs) also found in many RNA-binding proteins [19]. This region contains a short sequence rich in arginine and lysine residues, TRKQAR, which is characteristic of ARMs. However, since this motif is not sufficient by itself to ensure RNA binding, additional amino acids present in the 33-amino acid segment are required for specific contacts with RNA. Likewise, three N-terminal argininerich motifs, termed R1, R2 and RS, are required for the RNA-binding activity of UL69 [20]. Both R1 and R2 show similarity with ARMs which have been identified in number of viral RNA-binding proteins and may function as a bipartite ARM. Removal of RS in combination with either R1 or R2 abolishes the RNA-binding

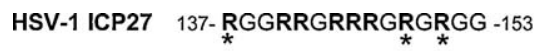 \\ HCMV UL69 16- LSERERRARRARRFCLDYEPVPRKFRRERSPTSPSTRN GAAASEYHLAEDTVGAASHHHRPCVPARRPRYSKDDDT EGDPDHYPPPLPPSSRHALGGTGGHIIMGTAGFRGGHR ASSSFKRRV -140}

EBV EB2 189-RSTRKQARQERSQRPLPNKPWFDMSLVKPVSKIT -224

VZV IE4 70- RYSKRRHGRRESYHHNRPKTLVVVLPDSNHHGGRDVET GYARIERGHRRSSRSYNTQSSRKHRDRSLSNRRRRPTTP PAMTTGERNDQTHDESYRLRFSKRDARRERIRK - 181

Fig. 4. Comparison of the different RNA-binding domains (RBDs) sequences. The HSV1 ICP27 RNA-binding domain is constituted by a RGG box. All arginine residues are indicated in bold and the three methylated arginine residues are marked by asterisks. The HCMV UL69 RNA-binding domain contains three arginine-rich (indicated in bold) motifs, termed R1, R2 and RS (underlined). The EBV EB2 RNA-binding domain is an arginine-rich (indicated in bold) region that contains a short sequence (underlined) characteristic of ARMs. The VZV IE4 RNA-binding domain maps to the three argininerich (indicated in bold) motifs, termed Ra, Rb and Rc (underlined). Numbers refer to the positions of the amino acid sequences within each protein. activity of UL69 both in vitro and in vivo. Recently, we have shown that IE4 is able to bind directly to RNA in vitro and the three Nterminal arginine-rich domains $\mathrm{Ra}, \mathrm{Rb}$ and $\mathrm{Rc}$ of IE4 were implicated in this interaction [3].

Although one might assume that protein domains with numerous arginine residues bind non-specifically to RNA, sequence-specific interactions were demonstrated for the majority of the ARM-containing proteins investigated so far which includes the HIV-1 proteins REV and TAT or the phage $\lambda \mathrm{N}$ protein. Although there is no evidence for sequence-specific RNA-binding by ICP27, EB2 and UL69 in vitro $[17,19,20]$, several data suggest the existence of a mechanism that confers specificity for the recognition of RNA in vivo (reviewed in Ref. [21]). Likewise, although the RNA-binding specificity of IE4 is still to be further explored, preliminary data in infection suggest that the mechanism could be transcript- or gene class-dependent [3]. Since all these herpesviral mRNA export factors interact with several viral and cellular proteins, it is conceivable that some of these interactions may be needed to confer specificity to RNA recognition. In addition, the secondary structure of RNA may be crucial for its correct recognition.

\section{Their interactions with cellular mRNA export factors}

In metazoans, mature and well-processed mRNPs are transported into the cytoplasm by the essential export factor TAP/NXF1. This mRNA export receptor shuttles between the nucleus and the cytoplasm, and escorts competent mRNPs out of the nucleus through direct interaction with nucleoporins lining the NPC [22]. Because of its low affinity for binding mRNAs, TAP/NXF1 requires adaptor proteins to interface with mature transcripts ready for export. So far, the best-characterized adaptor of TAP/NXF1 is the Aly/REF protein [23], whose recruitment to mRNAs was shown to depend on the presence of UAP56 [24]. However, the observation that Aly/REF is dispensable for mRNA export in both Drosophila and $C$. elegans suggests the existence of additional mRNA export adaptors $[25,26]$. The shuttling SR proteins have recently been proposed to act as adaptor of TAP/NXF1 and have been shown to promote export of both intronless [27] and intron-containing [28] mRNAs. Furthermore, SRp20 and 9G8 were shown to directly interact with TAP/NXF1 $[28,29]$ in a phosphorylation-dependent manner [30,31].

Thus, besides their ability to bind RNA, the capacity of the herpesviral proteins to interact with factors of the cellular mRNA export machinery was also demonstrated. However, the way by which these proteins interfere with the cellular mRNA export pathway is not unique (Fig. 1).

In one hand, ICP27 and EB2 were shown to interact with the mRNA export receptor TAP/NXF1 and its adaptor Aly/REF. The Cterminal leucine-rich region of EB2 was shown to be implicated in the interaction with Aly/REF in vitro. Aly/REF co-immunoprecipitates also with EB2 from transfected cells through interaction with the same region, but this interaction is RNase-sensitive suggesting that in vivo these proteins are part of a ribonucleoprotein complex stabilized by RNA [9]. This Aly/REF binding motif was shown to be required for both EB2-mediated mRNA export and production of infectious virions. In addition to its indirect recruitment via Aly/ REF, TAP/NXF1 was also shown to directly interact with the Nterminal NES of EB2, containing several arginine-rich domains that cooperate to allow efficient interaction with TAP/NXF1 [32]. On its side, ICP27 was reported to interact with TAP/NXF1 either directly or through its interaction with Aly/REF. In infected cells, ICP27 was shown to relocate Aly/REF from sites of cellular splicing to sites of viral transcription [16]. This interaction with Aly/REF was first shown to occur through the ICP27 region that contains the RNAbinding domain [15], and was later demonstrated to be RNasesensitive, confirming that RNA also stabilizes the complex [9]. 
Direct interaction with TAP/NXF1 itself requires both ICP27 Nterminus and C-terminus [33], and was shown to be essential for both efficient export of viral transcripts and HSV-1 replication [34].

On the other hand, UL69 has not yet been shown to interact with Aly/REF or TAP/NXF1. Instead, UL69 was demonstrated to directly bind to the highly related cellular DEXD/H-box RNA helicases UAP56 and URH49 [20,35]. The interaction between UL69 and UAP56/ URH49 was demonstrated both in transfected and infected cells, and it was excluded that RNA acts as bridging factor since the complexes are RNase-insensitive. Therefore, in contrast to ICP27 and EB2, direct RNA-binding is dispensable for UL69 RNA export activity. Comparatively, both UAP56 interaction and nucleocytoplasmic shuttling are prerequisites for this UL69 function [20].

In the case of IE4, we showed that the protein interacts in vivo with the three shuttling SR proteins SRp20, 9G8 and ASF/SF2 [3]. This interaction, which is not bridged by RNA, was shown to occur through the N-terminal arginine-rich domains of IE4. Contrastingly, we showed that the recruitment of TAP/NXF1 by IE4 is RNasesensitive, which suggests that it occurs through the SR proteins binding and that the presence of RNA stabilizes these complexes.

Thus, although specificities exist, all the data suggest that the herpesviral mRNA export factors use a general mechanism in which they bind to specific viral mRNAs and recruit these transcripts to the cellular mRNA export TAP/NXF1 pathway through direct interaction with the receptor TAP/NXF1 or with one of its adaptors such as Aly/REF, UAP56/URH49 or the shuttling SR proteins.

\section{Their functions in the RNA export}

Very early, ICP27-mediated gene regulation was suggested to occur post-transcriptionally at the level of RNA processing [36]. Few years later, ICP27 was suggested to be involved in the nucleocytoplasmic RNA transport of virus intron-containing transcripts [37]. It was then demonstrated that ICP27 binds specifically to viral intronless mRNAs in both cellular compartments [38], indicating a direct role in the nuclear export of viral mRNAs. The shuttling of ICP27 has been also reported to mediate the expression of some viral late genes [39]. During the last 10 years, the role of ICP27 in RNA export has been extensively documented (reviewed in Refs. $[40,41]$ ). Recently, a microarray analysis has demonstrated that efficient export of the majority of viral transcripts requires that ICP27 be able to bind RNA and to interact with TAP/NXF1 [42]. Furthermore, the knockdown of TAP/ NXF1 was shown to result in barely detectable level of two late proteins $\mathrm{gB}$ and $\mathrm{gC}$, and in greatly reduced HSV-1 viral titers [34]. Very recently, the knockdown of SRp20 and 9G8 was also shown to reduce the virus growth, suggesting that these factors could play a role as HSV-1 mRNAs export adaptors and contribute to the overall export efficiency during HSV-1 infection [43].

Similarly, EB2 has been described to lead to an increased cytoplasmic accumulation of many mRNAs, including those from reporter genes and those from a subset of EBV early genes coding for essential proteins involved in the EBV DNA replication during the productive cycle [44]. Furthermore, EB2 has been shown to be essential for the production of infectious viral particles [45] and for the nuclear export of most, but not all, late mRNAs produced from genes implicated in the capsid assembly and maturation [46].

In contrast, while the required domains of UL69 and its cellular partners begin to be well defined, its RNA export activity has been only visualized in reporter assays $[20,35]$ and the viral targets of UL69 are still to be defined. Alternatively, since UL69 is also a constituent of HCMV virions, RNA-binding by UL69 might be involved in the packaging of RNAs into HCMV viral particles, which incorporate a subset of viral and cellular transcripts in the course of infection.
Recently, we showed that IE4 also influences export of a reporter mRNA and that the knockdown of TAP/NXF1 prevents this IE4-mediated export [3]. Furthermore, we observed that TAP/NXF1 is also required for the correct expression of some VZV genes involved in virions assembly. Thus, although the viral targets have to be further characterized, we suggested that IE4, through its interaction with the SR proteins, is able to access the export TAP/ NXF1 pathway to carry some specific VZV mRNAs to the cytoplasm.

\section{Their functions in the RNA alternative splicing}

ICP27 was previously shown to post-transcriptionally stimulate mRNA accumulation from a transfected viral late gene encoding the glycoprotein $\mathrm{C}(\mathrm{gC})$ [47]. Further studying this observation, it was observed that the gC mRNA produced by ICP27 transactivation is an unspliced form, due to the retention of a previously undescribed intron from the gC transcript [48]. Contrastingly, during a viral infection, both transcripts and both proteins are detected, and it appears that HSV-1 has evolved to express two versions of the $\mathrm{gC}$ transcript. The spliced transcript encodes a variant of the glycoprotein $C$ that lacks its transmembrane domain and that is efficiently secreted from infected cells, suggesting that it could function as a virulence factor. As ICP27 has been previously shown to inhibit cellular mRNA splicing during infection, this gC intron retention function could simply reflect a non-specific suppression of splicing. Alternatively, this ability of ICP27 may be a transcript- or intron-specific effect and not the result of a general inhibition of mRNA splicing. Although still to be confirmed, the gC gene could harbour a silencing element in its $5^{\prime}$ half, distinct from the intron, and the effects of this sequence could be overcome by ICP27. The same authors also showed that IE4 lacks this ICP27 capacity of alternative splicing and is not able to promote HSV-1 gC intron retention [48].

Also very recently, EB2 was shown to induce alternative splicing of a cellular RNA transcript, suggesting that viruses may also regulate cellular gene expression at the level of alternative splicing. As mentioned above, when expressed in EBV-negative cells, EB2 has a broadly inhibitory effect on cellular mRNA accumulation. Nevertheless, EB2 causes several cellular transcripts to accumulate at higher levels [49]. These transcripts include STAT1 and several interferon (IFN)-stimulated genes. The STAT1 protein is a central mediator of both type I (IFN- $\alpha / \beta)$ and type II (IFN- $\gamma$ ) IFN signal transduction pathways. It is expressed as two isoforms, STAT1 $\alpha$ and STAT1 $\beta$. STAT1 $\beta$ mRNA is generated by cleavage and polyadenylation at an alternative site in the last intron of the STAT1 pre-mRNA, and, as the produced protein lacks the transactivating domain encoded in the last exon, STAT1 $\beta$ acts as a dominant-negative repressor of STAT1 $\alpha$. EB2 was shown to disproportionately increase the relative amount of STAT $1 \beta$ mRNA [49]. More precisely, EB2 was identified to induce the alternative splicing of STAT1 at a novel $5^{\prime}$ splice site (Alt $5^{\prime} \mathrm{SS}$ ), resulting in a STAT $1 \alpha^{\prime}$ mRNA incapable of producing STAT1 $\alpha$ (Fig. 5A) [50]. EB2 was shown to act as a splicing factor by directly binding to the same STAT1 RNA region as ASF/SF2 and to compete with this splicing factor in the splice site selection. By increasing the STAT $1 \beta /$ STAT $1 \alpha$ RNA ratio, EB2 changes the relative levels of ISGs depending on how responsive they are to STAT1 $\beta$, which affects cellular apoptosis and resistance to viral infection. As we showed that IE4 directly interacts with ASF/SF2, we here wondered if IE4 also shares this novel EB2 function. We used the STAT1 minigene construct for in vivo splicing assay (Fig. 5B). HeLa cells were cotransfected with this reporter plasmid and an IE4- or its homologues-encoding vector. Total RNAs were extracted and STAT1 transcripts were observed by RT-PCR using specific primers amplifying both alternatively spliced products $\left(\alpha\right.$ or $\left.\alpha^{\prime}\right)$. We showed that EB2 is the unique homologue able to alternatively 

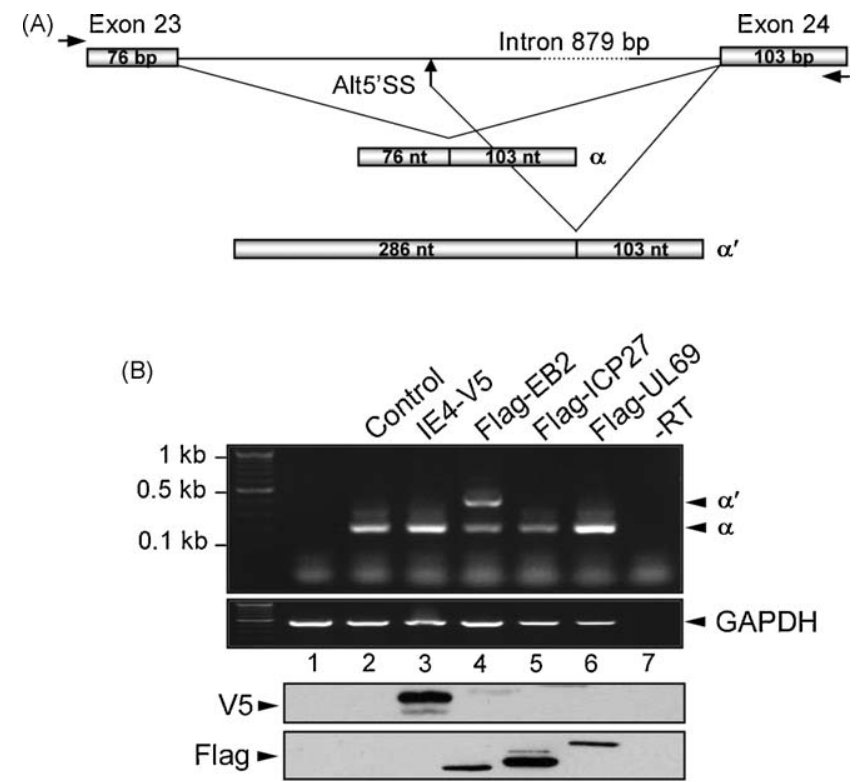

Fig. 5. IE4 and its homologues in the alternative splicing of the STAT1 RNA. (A) Schematic representation of the STAT1 minigene (adapted from Ref. [50]) which contains STAT1 exon 23, exon 24 and intervening sequence. Alternative processing events generate either the $\alpha$ or the $\alpha^{\prime}$ spliced form, in which the $\alpha 5^{\prime}$ donor site is skipped and the $\alpha^{\prime} 5^{\prime}$ donor site is used (Alt5'SS). (B) HeLa cells were transfected with the STAT1 minigene and expression plasmids as indicated (lanes 2-6). Untransfected cells were used as a negative control (lane 1). Total RNAs were isolated from transfected HeLa cells by TriPure/chloroform (Roche) extraction and isopropanol precipitation. A DNase treatment with DNase I recombinant RNase free (Roche) was performed according to the manufacturer's protocol to digest any DNA contamination from RNA preparations. Reverse transcription was performed with $1 \mu \mathrm{g}$ of purified total RNAs by use of M-MLV Reverse Transcriptase and Random Primers (Invitrogen) for $1 \mathrm{~h}$ at $37^{\circ} \mathrm{C}$. PCRs were performed on $2 \mu \mathrm{l}$ of cDNAs using GoTaq DNA polymerase (Promega) and specific primers (STAT1_FW 5'TGGAAAGCGTACACAATGTGT- ${ }^{\prime}$ and STAT1_RV 5'-CATACTGTCGAATTCTACAGA$3^{\prime}$ ) amplifying both alternatively spliced products $\left(\alpha\right.$ or $\left.\alpha^{\prime}\right)$. The PCR-amplified products were then analysed on $1 \%$ agarose gel and visualized by ethidium bromide staining. Reverse transcription without reverse transcriptase (RT, lane 7) was performed as purity control of our RNAs and GAPDH PCR was used as input control (GAPDH_FW 5'-CATCACTGCCACCCAGAAGACTGTGGA-3' and GAPDH_RV 5' TACTCCTTGGAGGCCATGTAGGCCATG- $3^{\prime}$ ). The expression of the different proteins was verified by Western blotting using anti-V5 and anti-Flag antibodies.

splice this STAT1 gene, and that the presence of either IE4, or ICP27 and UL69, only leads to the constitutively spliced STAT1 mRNA.

\section{Their regulation by phosphorylation}

As these four proteins are known to be phosphoproteins, it had been speculated that their activities could be regulated through phosphorylation. Very recently, two studies simultaneously highlighted a link between the cellular CDKs activity and the UL69 localization and functionality [51,52]. Furthermore, the HCMV protein kinase UL97, which had been characterized as a CDK-related kinase, was also shown to modulate the nuclear localization and activity of UL69. UL69 was shown to physically interact with UL97 and to co-localize with CDK9. In addition, UL69 acts as a specific substrate in in vitro kinase reactions with the viral kinase UL97 and all the CDKs analyzed (CDK-1, -2, -7 and -9). Interaction studies showed that UL97 interacts with the UL69 Nterminus, which consists of multiple overlapping functionally important regions, such as the NLS, the UAP56/URH49 interaction motif and the RNA binding site, as well as with the UL69 Cterminus, which contains the NES. Therefore, phosphorylation within these domains may trigger a fine-regulation of the distinct properties of UL69. Indeed, UL97-mediated phosphorylation of UL69 was demonstrated to enhance the latter's mRNA export activity, and protein kinase inhibitors, such as Gö6976 (UL97) and roscovitine or R58 (CDKs), significantly reduce this activity. Thus, cellular CDKs and viral UL97 activities are required to modulate the intranuclear localization and mRNA export function of UL69 during HCMV replication.

Similarly, it has been shown that the protein kinase CK2 $\alpha$ and $\beta$ subunits interact in vitro both individually, and more efficiently as a complex, with EB2 N-terminal and C-terminal domains [53]. Furthermore, EB2 was shown to co-immunoprecipitate from living cells with CK2. This latter was also shown to phosphorylate several residues within EB2 N-terminus, including the Ser-55, -56, and -57, which localize next to the EB2 NES. The phosphorylation mutant EB2S3A was shown to be unable to trans-complement EB2 in cells harboring an EB2-defective EBV genome. This defect is not due to an impaired nucleocytoplasmic shuttling but associated with a decreased cytoplasmic accumulation of several late viral mRNAs. These results suggest that the CK2-mediated EB2 phosphorylation is crucial for the production of EBV infectious virions.

ICP27 has also been demonstrated to be phosphorylated on serine residues during infection. Two serine residues at positions 16 and 18 within the leucine-rich $\mathrm{N}$-terminal region required for export were shown to be phosphorylated by CK2, and a serine at position 114 within the NLS was identified to be targeted by PKA [54]. As the S114A mutation diminishes the ICP27 import into the nucleus, it was suggested that the phosphorylation by PKA may regulate the ability of ICP27 to interact with importins. All ICP27 phosphorylation mutant viruses were also shown to reach lower growth curves compared to wild type HSV-1, further suggesting a role of phosphorylation in the various ICP27 functions during viral infection, including its shuttling and RNA export activities (reviewed in Ref. [41]). Recently, the ICP27 phosphorylation mutants at Ser-16, -18 , and -114 residues were shown to display altered functional interactions with Aly/REF and TAP/NXF1, although they are still able to bind HSV-1 mRNAs [55]. Interestingly, these mutants were also described to be defective in HSV-1 replication and viral gene expression [56].

SRPK1 is the main serine/threonine-protein kinase that specifically phosphorylates the SR proteins [57]. Through these phosphorylation events, SRPK1 regulates the localization and functions of the SR proteins, as well in constitutive and alternative splicing as in mRNAs export and translation (reviewed in Ref. [58]). As already mentioned, ICP27 was shown to interact with SRPK1, as well as with SRp20 and other SR proteins [59]. ICP27 is also phosphorylated by this kinase and several explanations for the ICP27-mediated SR protein hypo-phosphorylation have been suggested. ICP27 could simply compete with SR proteins for the SRPK1 binding, could mask potential phosphorylation sites in SR proteins, or could alter the SRPK1 conformation resulting in changes of its activity. These possibilities are not mutually exclusive and all result in splicing inhibition by impairing spliceosome assembly.

Similarly, we showed that IE4 interacts with SRPK1, mainly through its $\mathrm{Rb}$ domain [3]. We also showed that the Ser-136 residue located in this region is phosphorylated by the kinase. However, we were not able to demonstrate the role of this phosphorylation in the export activity of IE4. It could be implicated in the nuclear reimport of IE4, like it has been suggested for the SR proteins [30], or even be involved in the regulation of other still unknown functions of IE4. The in silico analysis of IE4 also predicts many phosphorylation sites for the CK2, PKA and PKC kinases, but no role of these potential phosphorylation events on the IE4 activities has been studied so far.

\section{The IE4-mediated recruitment of the TAP/NXF1 receptor}

SRp20 and 9G8 were shown to recruit TAP/NXF1 through a small arginine-rich peptide next to their RNA recognition motifs (RRMs) rather than through their C-terminal RS domains [29]. 


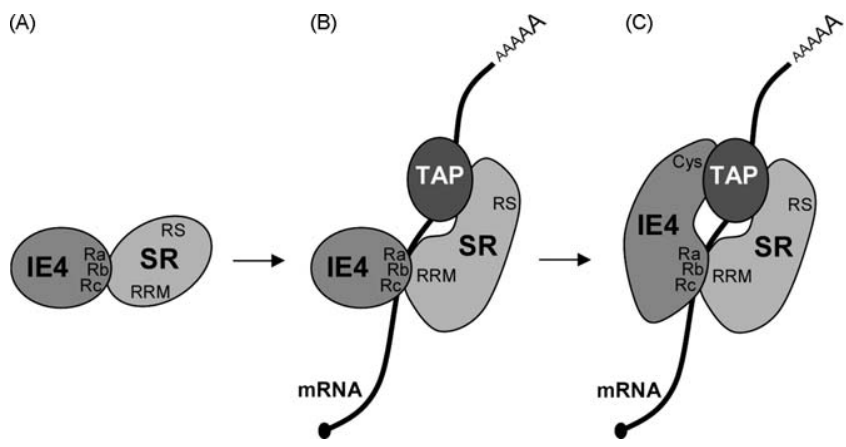

Fig. 6. Model of recruitment of TAP/NXF1 by IE4 in a SR proteins- and RNAdependent way. (A) In absence of RNA, IE4 and the SR proteins interact through the $\mathrm{Ra}, \mathrm{Rb}, \mathrm{Rc}$ domains of IE4 and a small peptide next to the RRMs of SR proteins. (B) In presence of RNA, a conformation change of the SR proteins allows the recruitment of TAP/NXF1. (C) IE4 binds TAP/NXF1 through its C-terminus leading to a tripartite export-competent complex.

Although it was not determined, it is convincible to think that, in absence of RNA, the interaction between IE4 and the SR proteins occurs through the Ra, Rb, Rc domains of IE4 and this small peptide (Fig. 6A). By contrast, in presence of RNA, the RNA-binding domains of these proteins would preferentially interact with the transcript, leading to a conformation change of the SR proteins and allowing the small peptide to recruit TAP/NXF1 (Fig. 6B). Interestingly, we showed that the C-terminal cystein-rich region of IE4 is also required for its export activity since the IE $4 \Delta$ cys mutant was shown to be incompetent in a reporter export assay [3]. Since IE4 seems to dimerize through this C-terminal region, the dimerization of IE4 could be a prerequisite to its function. However, it was shown that ICP27 interacts with TAP/NXF1 via its C-terminus, which contains a zinc finger-like domain (CCHC) [33]. Such a potential zinc finger domain is also present in the Cterminal region of IE4 [7]. We could thus hypothesize that the presence of RNA and the recruitment of TAP/NXF1 by the SR proteins would allow the C-terminal part of IE4 to bind TAP/NXF1, and that only these tripartite complexes would be competent for export (Fig. 6C). This model would support our data showing that the recruitment of TAP/NXF1 by IE4 is RNase-sensitive. Furthermore, IE4 was shown to be essential for VZV replication and more precisely the GKYFKC motif contained in the IE4 C-terminal region was shown to be crucial [60]. Thus, the role of IE4 as export adaptor and its capacity to access the TAP/NXF1 pathway could be indispensable for the VZV infection.

\section{IE4 and its homologues as alternative targets in antiherpetic therapies}

So far, majority of the antiherpetic agents are based on modified nucleoside analogues such as the currently available drugs acyclovir and gancyclovir. These drugs are competitively incorporated into viral DNA, what further blocks the DNA polymerase activity and inhibits the viral replication. However, over the past few years, the long-term usage of these antiviral agents led to the emergence of nucleoside-analogue-resistant herpesvirus strains, resulting in ineffective conventional therapies (reviewed in Ref. [61]). Additional antiherpetic treatments, such as various antisense-mediated strategies, are therefore under development, in order to specifically knockdown expression of some viral proteins.

On the basis of their crucial roles in the infectious cycle, the viral export factors described here must be considered as targets in these novel therapeutic approaches. Recently, the antiviral activity of peptide-conjugated phosphorodiamidate morpholino oligomers (PPMO) was investigated in HSV-1 ocular infection [62]. PPMO targeting ICP27 were shown to inhibit viral replication both in cell cultures and in eyes of mice. Since the antisense technique is in theory unlimited and that these PMO present pharmacological advantages compared to other antisense oligonucleotides, this novel technology could be further developed as alternative antiherpetic therapy.

In addition to target specific viral proteins, several recent studies suggest to exploit the cellular proteins as potential targets for the development of novel antiviral agents. Targeting a host factor may be a useful tool to avoid the emergence of mutant resistant strains. However, inhibition of multifunctional cellular proteins could more largely deregulate the host cell signalling pathways and might result in significant undesirable effects. In the mRNA export pathway, rather than targeting the essential TAP/ NXF1 receptor, the safest strategy would be to knockdown UAP56 or the shuttling SR proteins, which are the specific cellular proteins targeted by UL69 or IE4 in HCMV and VZV infection respectively $[3,35]$. Similarly, the specific knockdown of the DEAD-box RNA helicase DDX3 was shown to inhibit HIV-1 replication without affecting cell viability [63].

In addition to inhibit expression of these viral export factors, or expression of their cellular partners, antiviral drugs targeting their specific functions and regulation could also be developed. As all these proteins are regulated by phosphorylation, kinase inhibitors are promising key agents in antiviral therapies. Thus, similarly to roscovitine that was shown to reduce CDKs-mediated UL69 activity [51], specific inhibitors of CK2 or SRPK1 could potentially reduce ICP27, IE4 or EB2 export functions. Furthermore, viral kinases also represent potential targets in antiherpetic treatments. For example, maribavir, a selective inhibitor of the UL97 kinase [64], is currently under phase III clinical trial to treat HCMV infections.

Finally, example could be taken from the prototypical viral export factor HIV-1 REV protein, which has already been targeted in clinical trial. Indeed, in the anti-HIV gene-therapy study, the lentiviral vector transduction of a dominant-negative REV protein was shown to potently inhibit HIV-1 replication [65]. Thus, various mutants of the herpesviral export factors, impaired in their capacity of either nucleocytoplasmic shuttling, RNA-binding, or cellular proteins interaction, could be tested in their ability to act as dominant-negative inhibitors, thus corresponding to good candidates of protein-based agents in alternative antiherpetic therapies.

\section{Acknowledgments}

This work was supported by grants from the Belgian National Fund for Scientific Research (FNRS, Brussels, Belgium), the 'Communauté française' (Brussels, Belgium), the 'Leon Fredericq' Foundation (University of Liège, Belgium) and the IAP6/18 program from the Belgian Science Policy (University of Liège, Belgium). We thank Dr. S. Swaminathan (University of Florida, Gainesville, USA) for the kind gift of the STAT1 minigene.

\section{References}

[1] Davison AJ, Scott JE. The complete DNA sequence of varicella-zoster virus. J Gen Virol 1986;67(Pt 9):1759-816.

[2] Winkler M, Rice SA, Stamminger T. UL69 of human cytomegalovirus, an open reading frame with homology to ICP27 of herpes simplex virus, encodes a transactivator of gene expression. J Virol 1994;68:3943-54.

[3] Ote I, Lebrun M, Vandevenne P, Bontems S, Medina-Palazon C, Manet E, et al. Varicella-zoster virus IE4 protein interacts with SR proteins and exports mRNAs through the TAP/NXF1 pathway. PLoS One 2009;4:e7882.

[4] Mears WE, Rice SA. The herpes simplex virus immediate-early protein ICP27 shuttles between nucleus and cytoplasm. Virology 1998;242:128-37.

[5] Boyle SM, Ruvolo V, Gupta AK, Swaminathan S. Association with the cellular export receptor CRM 1 mediates function and intracellular localization of Epstein-Barr virus SM protein, a regulator of gene expression. J Virol 1999; 73:6872-81. 
[6] Lischka P, Rosorius O, Trommer E, Stamminger T. A novel transferable nuclear export signal mediates CRM1-independent nucleocytoplasmic shuttling of the human cytomegalovirus transactivator protein pUL69. EMBO J 2001;20:727183.

[7] Baudoux L, Defechereux P, Rentier B, Piette J. Gene activation by Varicellazoster virus IE4 protein requires its dimerization and involves both the arginine-rich sequence, the central part, and the carboxyl-terminal cysteine-rich region. J Biol Chem 2000;275:32822-31.

[8] Mears WE, Lam V, Rice SA. Identification of nuclear and nucleolar localization signals in the herpes simplex virus regulatory protein ICP27. J Virol 1995; 69:935-47

[9] Hiriart E, Farjot G, Gruffat H, Nguyen MV, Sergeant A, Manet E. A novel nuclear export signal and a REF interaction domain both promote mRNA export by the Epstein-Barr virus EB2 protein. J Biol Chem 2003;278:335-42.

[10] Kudo N, Matsumori N, Taoka H, Fujiwara D, Schreiner EP, Wolff B, et al. Leptomycin B inactivates CRM1/exportin 1 by covalent modification at a cysteine residue in the central conserved region. Proc Natl Acad Sci U S A 1999;96:9112-7.

[11] Chen L, Liao G, Fujimuro M, Semmes OJ, Hayward SD. Properties of two EBV Mta nuclear export signal sequences. Virology 2001;288:119-28.

[12] Farjot G, Buisson M, Duc Dodon M, Gazzolo L, Sergeant A, Mikaelian I. EpsteinBarr virus EB2 protein exports unspliced RNA via a Crm-1-independent pathway. J Virol 2000;74:6068-76.

[13] Phelan A, Clements JB. Herpes simplex virus type 1 immediate early protein IE63 shuttles between nuclear compartments and the cytoplasm. J Gen Virol 1997;78(Pt 12):3327-31.

[14] Soliman TM, Silverstein SJ. Herpesvirus mRNAs are sorted for export via Crm1dependent and -independent pathways. J Virol 2000;74:2814-25

[15] Koffa MD, Clements JB, Izaurralde E, Wadd S, Wilson SA, Mattaj IW, et al. Herpes simplex virus ICP27 protein provides viral mRNAs with access to the cellular mRNA export pathway. EMBO J 2001;20:5769-78.

[16] Chen IH, Sciabica KS, Sandri-Goldin RM. ICP27 interacts with the RNA export factor Aly/REF to direct herpes simplex virus type 1 intronless mRNAs to the TAP export pathway. J Virol 2002;76:12877-89.

[17] Mears WE, Rice SA. The RGG box motif of the herpes simplex virus ICP27 protein mediates an RNA-binding activity and determines in vivo methylation. J Virol 1996;70:7445-53.

[18] Souki SK, Sandri-Goldin RM. Arginine methylation of the ICP27 RGG box regulates the functional interactions of ICP27 with SRPK1 and Aly/REF during herpes simplex virus 1 infection. J Virol 2009;83:8970-5.

[19] Hiriart E, Bardouillet L, Manet E, Gruffat H, Penin F, Montserret R, et al. A region of the Epstein-Barr virus (EBV) mRNA export factor EB2 containing an arginine-rich motif mediates direct binding to RNA. J Biol Chem 2003;278:37790-

[20] Toth Z, Lischka P, Stamminger T. RNA-binding of the human cytomegalovirus transactivator protein UL69, mediated by arginine-rich motifs, is not required for nuclear export of unspliced RNA. Nucleic Acids Res 2006:34:1237-49.

[21] Toth Z, Stamminger T. The human cytomegalovirus regulatory protein UL69 and its effect on mRNA export. Front Biosci 2008;13:2939-49.

[22] Bachi A, Braun IC, Rodrigues JP, Pante N, Ribbeck K, von Kobbe C, et al. The Cterminal domain of TAP interacts with the nuclear pore complex and promotes export of specific CTE-bearing RNA substrates. RNA 2000;6:136-58.

[23] Stutz F, Bachi A, Doerks T, Braun IC, Seraphin B, Wilm M, et al. REF, an evolutionary conserved family of hnRNP-like proteins, interacts with TAP/ Mex67p and participates in mRNA nuclear export. RNA 2000;6:638-50.

[24] Luo ML, Zhou Z, Magni K, Christoforides C, Rappsilber J, Mann M, et al. PremRNA splicing and mRNA export linked by direct interactions between UAP56 and Aly. Nature 2001;413:644-7.

[25] Gatfield D, Izaurralde E. REF1/Aly and the additional exon junction complex proteins are dispensable for nuclear mRNA export. J Cell Biol 2002;159:579 88.

[26] Longman D, Johnstone IL, Caceres JF. The Ref/Aly proteins are dispensable for mRNA export and development in Caenorhabditis elegans. RNA 2003;9:88191.

[27] Huang Y, Steitz JA. Splicing factors SRp20 and 9G8 promote the nucleocytoplasmic export of mRNA. Mol Cell 2001;7:899-905

[28] Huang Y, Gattoni R, Stevenin J, Steitz JA. SR splicing factors serve as adapte proteins for TAP-dependent mRNA export. Mol Cell 2003;11:837-43.

[29] Hargous Y, Hautbergue GM, Tintaru AM, Skrisovska L, Golovanov AP, Stevenin $\mathrm{J}$, et al. Molecular basis of RNA recognition and TAP binding by the SR proteins SRp20 and 9G8. EMBO J 2006;25:5126-37.

[30] Huang Y, Yario TA, Steitz JA. A molecular link between SR protein dephosphorylation and mRNA export. Proc Natl Acad Sci U S A 2004;101:9666-70.

[31] Lai MC, Tarn WY. Hypophosphorylated ASF/SF2 binds TAP and is present in messenger ribonucleoproteins. J Biol Chem 2004;279:31745-9.

[32] Juillard F, Hiriart E, Sergeant N, Vingtdeux-Didier V, Drobecq H, Sergeant A et al. Epstein-Barr virus protein EB2 contains an N-terminal transferable nuclear export signal that promotes nucleocytoplasmic export by directly binding TAP/NXF1. J Virol 2009;83:12759-68.

[33] Chen IH, Li L, Silva L, Sandri-Goldin RM. ICP27 recruits Aly/REF but not TAP/ NXF1 to herpes simplex virus type 1 transcription sites although TAP/NXF1 is required for ICP27 export. J Virol 2005;79:3949-61.

[34] Johnson LA, Li L, Sandri-Goldin RM. The cellular RNA export receptor TAP NXF1 is required for ICP27-mediated export of herpes simplex virus 1 RNA, but the TREX complex adaptor protein Aly/REF appears to be dispensable. J Virol 2009;83:6335-46.
[35] Lischka P, Toth Z, Thomas M, Mueller R, Stamminger T. The UL69 transactivator protein of human cytomegalovirus interacts with DEXD/H-Box RNA helicase UAP56 to promote cytoplasmic accumulation of unspliced RNA. Mol Cell Biol 2006;26:1631-43.

[36] Sandri-Goldin RM, Mendoza GE. A herpesvirus regulatory protein appears to act post-transcriptionally by affecting mRNA processing. Genes Dev 1992; 6:848-63.

[37] Phelan A, Dunlop J, Clements JB. Herpes simplex virus type 1 protein IE63 affects the nuclear export of virus intron-containing transcripts. J Virol 1996;70:5255-65.

[38] Sandri-Goldin RM. ICP27 mediates HSV RNA export by shuttling through a leucine-rich nuclear export signal and binding viral intronless RNAs through an RGG motif. Genes Dev 1998;12:868-79.

[39] Soliman TM, Sandri-Goldin RM, Silverstein SJ. Shuttling of the herpes simplex virus type 1 regulatory protein ICP27 between the nucleus and cytoplasm mediates the expression of late proteins. J Virol 1997;71:9188-97.

[40] Smith RW, Malik P, Clements JB. The herpes simplex virus ICP27 protein: a multifunctional post-transcriptional regulator of gene expression. Biochem Soc Trans 2005;33:499-501.

[41] Sandri-Goldin RM. The many roles of the regulatory protein ICP27 during herpes simplex virus infection. Front Biosci 2008;13:5241-56.

[42] Johnson LA, Sandri-Goldin RM. Efficient nuclear export of herpes simplex virus 1 transcripts requires both RNA binding by ICP27 and ICP27 interaction with TAP/NXF1. J Virol 2009;83:1184-92.

[43] Escudero-Paunetto L, Li L, Hernandez FP, Sandri-Goldin RM. SR proteins SRp20 and 9G8 contribute to efficient export of herpes simplex virus 1 mRNAs. Virology 2010;401:155-64.

[44] Ruvolo V, Gupta AK, Swaminathan S. Epstein-Barr virus SM protein interacts with mRNA in vivo and mediates a gene-specific increase in cytoplasmic mRNA. J Virol 2001:75:6033-41.

[45] Gruffat H, Batisse J, Pich D, Neuhierl B, Manet E, Hammerschmidt W, et al. Epstein-Barr virus mRNA export factor EB2 is essential for production of infectious virus. J Virol 2002;76:9635-44.

[46] Batisse J, Manet E, Middeldorp J, Sergeant A, Gruffat H. Epstein-Barr virus mRNA export factor EB2 is essential for intranuclear capsid assembly and production of gp350. J Virol 2005;79:14102-11.

[47] Perkins KD, Gregonis J, Borge S, Rice SA. Transactivation of a viral target gene by herpes simplex virus ICP27 is posttranscriptional and does not require the endogenous promoter or polyadenylation site. J Virol 2003;77:9872-84.

[48] Sedlackova L, Perkins KD, Lengyel J, Strain AK, van Santen VL, Rice SA. Herpes simplex virus type $1 \mathrm{ICP} 27$ regulates expression of a variant, secreted form of glycoprotein C by an intron retention mechanism. J Virol 2008;82:7443-55.

[49] Ruvolo V, Navarro L, Sample CE, David M, Sung S, Swaminathan S. The EpsteinBarr virus SM protein induces STAT1 and interferon-stimulated gene expression. J Virol 2003;77:3690-701.

[50] Verma D, Swaminathan S. Epstein-Barr virus SM protein functions as an alternative splicing factor. J Virol 2008;82:7180-8.

[51] Rechter S, Scott GM, Eickhoff J, Zielke K, Auerochs S, Muller R, et al. Cyclindependent kinases phosphorylate the cytomegalovirus RNA export protein pUL69 and modulate its nuclear localization and activity. J Biol Chem 2009;284:8605-13.

[52] Thomas M, Rechter S, Milbradt J, Auerochs S, Muller R, Stamminger T, et al. Cytomegaloviral protein kinase pUL97 interacts with the nuclear mRNA export factor pUL69 to modulate its intranuclear localization and activity. J Gen Virol 2009;90:567-78.

[53] Medina-Palazon C, Gruffat H, Mure F, Filhol O, Vingtdeux-Didier V, Drobecq H, et al. Protein kinase CK2 phosphorylation of EB2 regulates its function in the production of Epstein-Barr virus infectious viral particles. J Virol 2007; 81:11850-6.

[54] Zhi Y, Sandri-Goldin RM. Analysis of the phosphorylation sites of herpes simplex virus type 1 regulatory protein ICP27. J Virol 1999;73:3246-57.

[55] Corbin-Lickfett KA, Rojas S, Li L, Cocco MJ, Sandri-Goldin RM. ICP27 phosphorylation site mutants display altered functional interactions with cellular export factors Aly/REF and TAP/NXF1 but are able to bind herpes simplex virus 1 RNA. J Virol 2010:84:2212-22.

[56] Rojas S, Corbin-Lickfett KA, Escudero-Paunetto L, Sandri-Goldin RM. ICP27 phosphorylation site mutants are defective in herpes simplex virus 1 replication and gene expression. J Virol 2010;84:2200-11.

[57] Gui JF, Tronchere H, Chandler SD, Fu XD. Purification and characterization of a kinase specific for the serine- and arginine-rich pre-mRNA splicing factors. Proc Natl Acad Sci U S A 1994;91:10824-8.

[58] Long JC, Caceres JF. The SR protein family of splicing factors: master regulators of gene expression. Biochem J 2009;417:15-27.

[59] Sciabica KS, Dai QJ, Sandri-Goldin RM. ICP27 interacts with SRPK1 to mediate HSV splicing inhibition by altering SR protein phosphorylation. EMBO J 2003;22:1608-19.

[60] Sato B, Sommer M, Ito H, Arvin AM. Requirement of varicella-zoster virus immediate-early 4 protein for viral replication. J Virol 2003;77:12369-72.

[61] Siakallis G, Spandidos DA, Sourvinos G. Herpesviridae and novel inhibitors. Antivir Ther 2009;14:1051-64

[62] Moerdyk-Schauwecker M, Stein DA, Eide K, Blouch RE, Bildfell R, Iversen P, et al. Inhibition of HSV-1 ocular infection with morpholino oligomers targeting ICP0 and ICP27. Antiviral Res 2009;84:131-41.

[63] Ishaq M, Hu J, Wu X, Fu Q, Yang Y, Liu Q, et al. Knockdown of cellular RNA helicase DDX3 by short hairpin RNAs suppresses HIV-1 viral replication without inducing apoptosis. Mol Biotechnol 2008;39:231-8. 
[64] Biron KK, Harvey RJ, Chamberlain SC, Good SS, Smith 3rd AA, Davis MG, et al. Potent and selective inhibition of human cytomegalovirus replication by $1263 \mathrm{~W} 94$, a benzimidazole L-riboside with a unique mode of action. Antimicrob Agents Chemother 2002;46:2365-72.
[65] Bahner I, Sumiyoshi T, Kagoda M, Swartout R, Peterson D, Pepper K, et al. Lentiviral vector transduction of a dominant-negative Rev gene into human CD34+ hematopoietic progenitor cells potently inhibits human immunodeficiency virus-1 replication. Mol Ther 2007;15:76-85. 